\title{
Recurrence quantification analysis as a tool for characterization of non-linear mesocosm dynamics
}

\author{
Joachim W. Dippner ${ }^{1, *}$, Reinhard Heerkloss ${ }^{2}$, Joseph P. Zbilut ${ }^{3}$ \\ ${ }^{1}$ Institut für Ostseeforschung, Warnemünde, Seestr. 15, 18119 Rostock, Germany \\ ${ }^{2}$ Institut für Aquatische Biologie, Universität Rostock, Albert-Einstein-Str. 3, 18051 Rostock, Germany \\ ${ }^{3}$ Department of Molecular Biophysics and Physiology, Rush Medical College, 1653 W. Congress, Chicago, Illinois 60612, USA
}

\begin{abstract}
A mesocosm experiment was performed in order to observe the behaviour of an aquatic community under stable external conditions. The hypothesis was that the community would show temporal fluctuations that correspond to the principles of deterministic chaos. Water from a brackish (9 psu) location was kept from 31 March 1989 to 11 July 1990 in a 1001 mesocosm under artificial laboratory conditions to develop a species-depleted climax community of plankton. The mesocosm was reared for a further period of 1764 d (12 July 1990 to 11 May 1995) under controlled light and temperature conditions and sampled in short intervals of 3 to $4 \mathrm{~d}$. During the period of observations there were no changes in species composition, but strong fluctuation in biomass occurred. The biomass data for picophytoplankton, nanophytoplankton, copepods and rotifers were used to check the systems dynamics for chaotic behaviour by recurrence quantification analysis (RQA), a new method which is applied here to analyse non-linear dynamics in marine ecosystems for the first time. Recurrence histograms show a single period of $7 \mathrm{wk}$ of picophytoplankton, a broad range of periods for nanophytoplankton between 4 and $7 \mathrm{wk}$ with isolated peaks at 5 and $7 \mathrm{wk}$, a broad range of periods for copepods with a peak at $5 \mathrm{wk}$ and 2 minor peaks at 3 and $12.5 \mathrm{wk}$, and transient behaviour in rotifers with peaks at 5.5 and $11.5 \mathrm{wk}$, with non-linear interaction between selected bands of periods. A comparison with shuffled time series shows that the system dynamic is deterministic with transients and that a non-stationary trend exists in the phytoplankton time series. No chaotic behaviour could be detected.
\end{abstract}

KEY WORDS: Mesocosm $\cdot$ Recurrence quantification analysis $\cdot$ Non-linear dynamics $\cdot$ Chaos $\cdot$ Plankton

\section{INTRODUCTION}

Variation in the abundance of the different species forming natural communities is governed by external factors and internal interactions between species within the communities. It has been shown mathematically that the dynamics which arise when 2 or more species are interlinked only by internal interactions, i.e., under constant external conditions, may conform to the principles of deterministic chaos (May \& Oster 1976, Schaffer \& Kot 1985, Hastings \& Powell 1991,

*E-mail: joachim.dippner@io-warnemuende.de
Schaffer 1991, Solé \& Valls 1992, Allen 1993, Ascioti et al. 1993, Doveri et al. 1993, Hastings et al. 1993, Turchin 1993, McCann \& Yodzis 1994, Rinaldi \& Solidoro 1998). Aquatic ecologists became especially interested in cases of chaotic behaviour after Huismann \& Weissing (1999) reported their results. They investigated competition models of phytoplankton and could show that under conditions of strong oscillations chaos keeps species diversity high. Hutchinson (1961) introduced the problem of high diversity of planktonic communities in face of a quite limited number of limiting resources in rather homogeneous pelagic environment and called it 'paradoxon of plankton'. There are more species in the plankton than there should be according 
to the theory of competitive exclusion. On the other hand, the existence of chaos in ecological systems has been questioned by Joergensen (1997, p. 264) who concluded from energy models that for planktonic species, natural selection favours growth parameters that keep the communities away from chaos.

The results of multi-species models have not so far been adequately supported by empirical studies. Compared with other biological fields of application of chaos theory, the openness of ecological systems to external influences makes it difficult to interpret field data from large scale ecosystems. To overcome this difficulty an experiment with a plankton mesocosm under constant external conditions was performed. The present study describes a non-linear analysis of a dataset from this experiment. Details about the design of the mesocosm and a full set of the experimental data have been published previously (Heerkloss \& Klinkenberg 1998). Strong fluctuations in biomass values were observed looking quite irregular and non-periodic. Therefore, it was concluded in that paper that the observed fluctuations of the different species could be chaotic.

In the present paper a mathematical analysis of these data is performed to check the occurrence of chaos using a tool for non-linear analysis. The method used here is Recurrence (time correlation) Quantification Analysis (RQA), a relatively new method developed in theoretical physics (Eckmann et al. 1987, Zbilut \& Webber 1992) and applied to various non-linear problems such as heartbeat dynamics and irregularities (Giuliani et al. 1998a), molecular dynamics (Manetti et al. 1999), analysis of protein sequences (Giuliani et al. 2000, Zbilut et al. 1998a) etc. The RQA method is applied here for the first time to analyse non-linear dynamics in marine ecosystems.

\section{MATERIALS AND METHODS}

Mesocosm experiment. A cylindrical plastic container $(74 \mathrm{~cm}$ high and $45 \mathrm{~cm}$ in diameter) was filled with a $10 \mathrm{~cm}$ sediment layer and $90 \mathrm{l}$ of water from a near-shore station of the Barther Bodden (Darss-Zingst estuary, southern Baltic Sea $54^{\circ} 26^{\prime} \mathrm{N}, 12^{\circ} 42^{\prime}$ E) on 31 March 1989. It was kept at room temperature for the period from 31 March 1989 to 11 July 1990 to obtain a species depleted climax community of plankton. The mesocosm community was reared for a further period of 252 wk from 12 July 1990 to 11 May 1995 under constant light and temperature conditions and sampled twice a week. That results in a sampling period of 3 to $4 \mathrm{~d}$ with an average sampling rate of $3.5 \mathrm{~d}$. There was little change in species structure during that time but a strong fluctuation of biomass values and dominance structure. The conditions were kept constant: temperature $20^{\circ} \mathrm{C}$, light $50 \mu \mathrm{E} \mathrm{m}{ }^{-2} \mathrm{~s}^{-1}, 16: 8 \mathrm{~h}$ light:dark cycle, and constant aeration.

For sampling of nutrients and plankton during the whole period of $1764 \mathrm{~d}$, a total of $26 \mathrm{l}$ of water was removed and replaced by $0.45 \mu \mathrm{m}$ filtered and aged biotope water to compensate water losses due to evaporation and sampling. This procedure might have resulted in a pertubation of the system and it is unclear how the system dynamics would have been influenced; however, neither significant changes in species composition nor in dominance structure were observed. In addition, a further external impact might resulted from zooplankton sampling. Zooplankton was sampled by filtering $10 \mathrm{l}$ through a $50 \mu \mathrm{m}$ net. This corresponds to a removal of $22.2 \%$ of the standing stock per week. For our present study this impact is of minor importance, because no influence on the variability could be detected.

In the period from 23 November 1990 to 5 March 1991 the light condition was reduced from 16 to $12 \mathrm{~h} \mathrm{~d}^{-1}$ for a special experiment. The system reacted with a pronounced change in its dominance structure from phytoflagellates to picophytoplankton. After a return to the previous light condition the system also returned to the previous dominance structure. For the purpose of detecting chaos in a real ecological community, it was important to keep the external conditions constant. Due to the absence of a seasonal cycle in abiotic factors, the system must be considered as artificial compared to realistic conditions in nature. However, the goal of the mesocosm experiment was to create an empirical situation which is comparable to that of multi-species mathematical models. As a rule, these models are characterized by dynamics without changes in external conditions. Therefore, the period of reduced light and the period of return of the previous dominance structure (i.e. the period from 23 November 1990 to 17 June 1991) was removed from the original datasets, which results in a period of investigation of $1559 \mathrm{~d}$ containing a total of 446 data records for the analysis.

The development of the mesocosm has already been described by Heerkloss \& Klinkenberg (1998). Here we confine ourselves to 4 planktonic groups: picophytoplankton, consisting of 1 to $3 \mu \mathrm{m}$ unicellular coccoid cyanobacteria; nanophytoplankton, consisting mainly of 3 to $5 \mu \mathrm{m}$ eukaryontic flagellates; copepods, consisting of the filtering calanoid copepod Eurytemora affinis; and rotifers, consisting of the marine species Brachionus plicatis (Fig. 1).

Mathematical analysis. Recurrences: Recurrence times are certainly not new. Poincaré is perhaps the most famous for describing them in the context of dynamical systems as points which visit a small region 
of phase space (Bergelson 2000). The statistical literature also points out that recurrences are the most basic of relations (Feller 1968). In this respect, it is important to reiterate the fact that calculation of recurrence times, unlike other methods such as Fourier, Wigner-Ville or wavelets, requires no transformation of the data, and can be used for both linear and non-linear systems (Zbilut et al. 1992, Zbilut \& Webber 1992). Because recurrences are simply tallies, they make no mathematical assumptions. Given a reference point, $\mathbf{X}_{0}$, and a ball, $B_{r}$, of radius $r$, a point is said to recur if:

$$
B_{r}\left(\mathbf{X}_{0}\right)=\left\{\mathbf{X}:\left\|\mathbf{X}-\mathbf{X}_{0}\right\| \leq r\right\}
$$

A trajectory of size $\mathrm{N}$ falling within $B_{T}\left(\mathbf{X}_{0}\right)$ is denoted as:

$$
S_{1}=\left\{\mathbf{X}_{t_{1}}, \mathbf{X}_{t_{2}}, \ldots, \mathbf{X}_{t_{i} \cdots} \cdots\right\}
$$

with the recurrence times defined as:

$$
T_{1}(i)=\mathrm{t}_{i+1}-\mathrm{t}_{i,}, \quad i=1,2, \ldots \mathrm{N}
$$

If the dynamics is stationary and ergodic on an attractor, the reference point can be chosen arbitrarily. In most real life contexts this cannot be assumed, yet, as Eckmann et al. (1987) have pointed out, this very condition is revealed by contextual changes seen in a plot of recurrences.

Recurrence plots: Given a scalar time series $\{x(i)=$ $1,2,3, \ldots\}$ an embedding procedure will form a vector, $\mathbf{X}_{i}=(x(i), x(i+L), \ldots, x(i+(m-1) L))$ with $m$ the embedding dimension and $L$ the lag. $\left\{\mathbf{X}_{i}=1,2,3, \ldots, N\right\}$ then represents the multi dimensional process of the time series as a trajectory in m-dimensional space. Recurrence plots (RP) are symmetrical $N \times N$ arrays in which a point is placed at $(i, j)$ whenever a point $\mathbf{X}_{i}$ on the trajectory is close to another point $\mathbf{X}_{j}$. The closeness between $\mathbf{X}_{i}$ and $\mathbf{X}_{j}$ is expressed by calculating the Euclidian distance between these 2 normed vectors, i.e., by subtracting one from the other: $\left\|\mathbf{X}_{i}-\mathbf{X}_{j}\right\| \leq r$ where $r$ is a fixed radius. If the distance falls within this radius, the 2 vectors are considered to be recurrent, and graphically this can be indicated by a dot.

An important feature of such matrixes is the existence of short line segments parallel to the main diagonal, which correspond to sequences $(i, j),(i+1, j+1), \ldots$, $(i+k, j+k)$ such that the piece of $\mathbf{X}(j), \mathbf{X}(j+1), \ldots, \mathbf{X}(j+k)$, is close to $\mathbf{X}(i) \ldots, \mathbf{X}(i+1), \ldots, \mathbf{X}(i+k)$ in series which are deterministic. The absence of such patterns suggest randomness.

It is quite obvious that the recurrence plot depends strongly on 3 quantities, namely the lag $L$, the choice of the embedding dimension, and the selected radius $r$. To determine $L$, the autocorrelation function of the time series is computed. To avoid false linear correlations, the lag value is set to the first zero crossing of the autocorrelation function. Formally, it is not trivial to estimate the correct (minimal) embedding dimension of the attractor. If the embedding is done correctly, the system dynamic is invariant and certain properties of the original system are preserved in the embedded space. In theory, the embedding dimension can be estimated by computing the correlation dimension of the system (Schuster 1989); however, such calculations are problematic if the dataset is too short, noisy, or non-stationary. Hence, it is better to overestimate the embedding dimension in order to preserve the invariant system dynamic. Practically, the embedding dimension is estimated graphically (A. Giuliani pers. comm.) in the following way. One starts with a high embedding dimension of (e.g. 20), computes the recurrence plot with a minimal radius and compares the recurrence plot with the recurrence plot of the embedding dimension of 19. If no difference exists optically, the system dynamic is still invariant
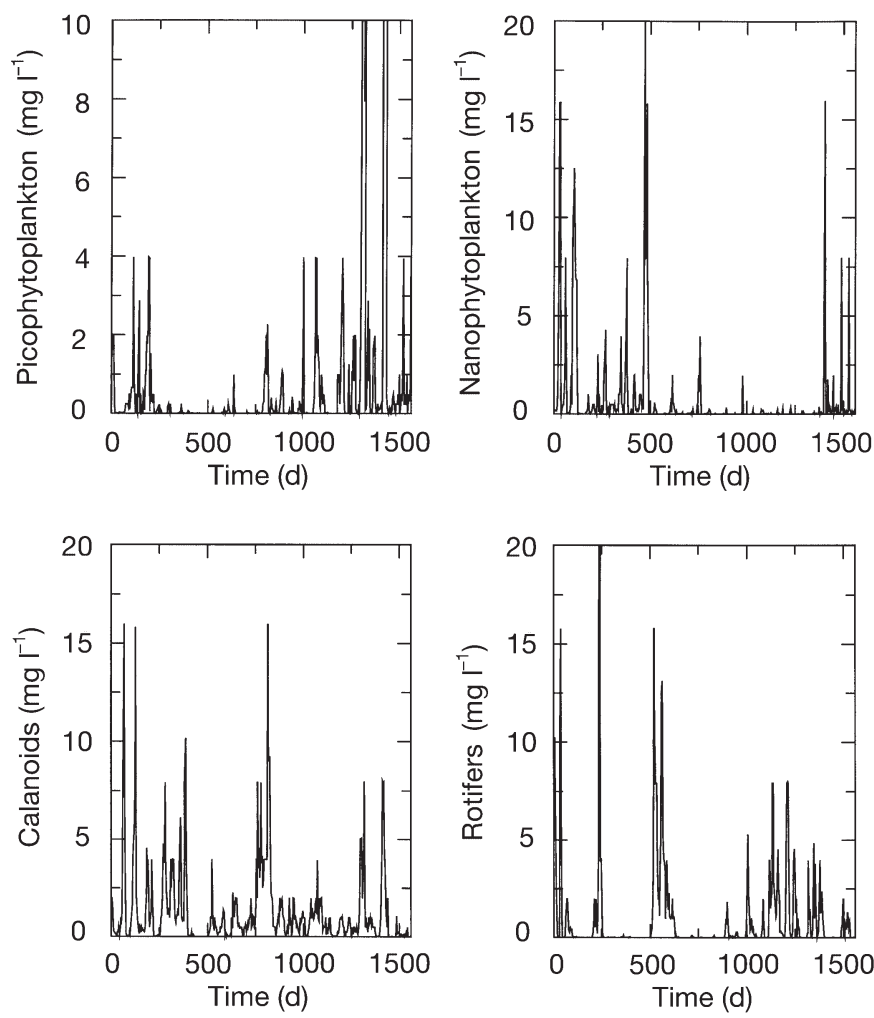

Fig. 1. Observed picophytoplankton, nanophytoplankton, calanoids and rotifers in fresh wt $\mathrm{l}^{-1}$ as a function of time. Composition of biomass: picophytoplankton consists of unicellular or filamentous cyanobacteria between 1 and $3 \mu \mathrm{m}$ in diameter, nanophytoplankton of autotrophic flagellates between 3 and $5 \mu \mathrm{m}$, calanoids of the genuine brackish water species Eurytemora affinis, and rotifers of the marine species Brachionus plicatilis. Peak values are for picophytoplankton $89.9 \mathrm{mg} \mathrm{l}^{-1}$ at Day 1318 and $63.9 \mathrm{mg} \mathrm{l}^{-1}$ at Day 1424, and $38 \mathrm{mg} \mathrm{l}^{-1}$ at Day 241 in rotifers 
and the procedure is continued with the comparison of dimension 19 with dimension 18 , and so on. The last dimension before the recurrence plot changes is graphically estimated as the 'best' embedding dimension. As a practical matter, in general, it is preferrable to maintain a relatively high embedding especially for possibly non-stationary dynamics: such dynamics may 'move' between different dimensions, and research has suggested that to maintain the essential dynamics 'over-embedding' is warranted (Hegger et al. 2000). To estimate the optimal radius in the distance matrix, the logarithm of the percent of recurrence is computed as function of the logarithm of the radius. From this graph the transition from the noise level to the signal space can be obtained, which will be shown later in an example.

Recurrence quantification: RQA was developed to provide quantification of important aspects revealed by the plot because graphical representation may be difficult to evaluate. Recurrent points which form diagonal line segments are considered to be deterministic, as distinguished from random points which form no patterns. Unfortunately, beyond general impressions of drift and determinism, the plots themselves provide no quantification. Zbilut \& Webber (1992) developed several strategies to quantify features of such plots originally pointed out by Eckmann et al. (1987).

This leads to the generation of five quantitative descriptors of the recurrence plot that are useful in the quantitative assessment of highly non-linear systems: the percent recurrence, the percent determinism, the Shannon entropy, the trend and the maximum diagonal line length maxline, which has been introduced as an approximation to the largest positive Lyapunov exponent. The percent recurrence quantifies the percentage of the plot occupied by recurrent points. The percent determinism quantifies the percentage of recurrent points which form upward diagonal line segments with a minimum of 2 adjacent points. These quantities are calculated by a straightforward summation of all actually occurring points. Entropy addresses the complexity of the recurrence plot by employing principles from Shannon's information entropy of the line length distribution (Shannon \& Weaver 1949). The more complex the deterministic structure, the larger the number of bits per bin required to represent the dynamic and the higher the entropy. The entropy is calculated using the histogram of line segment length according to Shannon's equation: $I=-\sum_{i=1}^{n} p_{i} \log _{2} p_{i}$, where $p_{i}$ are probabilities to find a system in a certain state, and $I$ is the information contained in the system. The trend is a measure of the paling of recurrent points away from the central diagonal and addresses the nonstationarity characteristics in recurrence plots. The trend is computed as the least-squares regression best fit through the points from the diagonal to the corner. Finally, maxline is the length of the longest line segment. The reciprocal of maxline is an approximation of the largest positive Lyapunov exponent and is a measure of system divergence (Trulla et al. 1996). These recurrence variables quantify the deterministic structure and complexity of the plot.

The data obtained can also be used to obtain estimations of local Lyapunov exponents, information entropy, or simply plotted as $N_{\text {recurrences }}$ vs period; i.e., a histogram of recurrence times. In the case of histograms, strictly periodic points demonstrate instrumentally sharp peaks, whereas chaotic or non-linear systems reveal more or less wider peaks depending upon the radius chosen and noise effects. RQA can also be combined with other statistical techniques such as principal components analysis in order to gain more information (Zbilut et al. 1998b, 2000, Gao 1999).

\section{RESULTS}

The original time series of the 4 selected plankton groups are shown in Fig. 1. Visually, it is nearly impossible to say anything about the system dynamics from these figures. No clear periods are visible and some strong peaks are followed by epochs with noisy structure. So, our first impression after of the time dependence of the signals was a possibly chaotic system dynamic. Therefore, RQA which also allows the analysis of chaotic behaviour, was applied to these datasets for an insight into the dynamics of the mesocosm. In all time series, the time lag of 6 time steps ( $3 \mathrm{wk}$ ) has been derived from the first minimum of the autocorrelation function. The fact that the autocorrelation function decays rapidly could be, qualitatively, a possible criterion for chaotic behaviour (Schuster 1989), but also for a system with a high level of white noise (Jenkins \& Watts 1968). From the autocorrelation function itself neither a conclusion with respect to chaotic behaviour nor to a noisy sytem can be drawn. To get an idea of possible dominant modes in the system, a empirical orthogonal function (EOF), also known as 'principle components' analysis was performed with the 4 time series. The amount of explained variance in the EOF analysis shows a nearly equal distribution of the 4 time series, indicating either white noise or 4 independent oscillatory eigenmodes. The autocorrelation functions as well as the EOF analysis indicate a relatively high noise level which is a crucial factor for pattern recognition and the ability to discriminate between signal and noise. The RQA allows the possibility of overcoming this separation problem (Giuliani et al. 1998b, Zbilut et al. $1998 b, c)$. 
The minimal embedding dimension of the attractor was graphically identified as 10 . The choice of this dimension also guarantees 'over-embedding', which is meaningful in non-stationary dynamics. Each time series has its own radius; however, all are very close together. The radius was chosen as $5 \mathrm{mg} \mathrm{l}^{-1}$ in the embedding space, large enough to capture signals rising above the noise floor. As an example, Fig. 2 shows the log of percentage recurrence as a function of the $\log$ radius for the calanoids. Up to a radius of $0.7(\log 5)$ the system is in the noise subspace. Then the signal subspace starts and if the radius is large enough and the logarithm of recurrence reaches a certain level all points on the attractor are recurrent. In this case, the results of the RQA are meaningless if the radius is set too high. The lowest number of upward diagonal recurrent points required to define a deterministic line is set to 3 points, which is short enough to identify possible chaotic behaviour.

Figs. 3 to 6 show the RQA of the 4 time series for 2 primary producers and 2 filtering consumer species, namely, pico- and nanophytoplankton, and calanoids and rotifers. The fundamental question is how to read the recurrent structure in the figures. A detailed description is given in Webber \& Zbilut (1994), which is briefly repeated here. Single isolated points are due to chance recurrence in the embedded dynamics. The apparent random scattering of recurrent points is attributed to high-dimensional stochastic processes. Upward diagonal lines result from patterns repeating themselves and indicate that deterministic rules are present in the system. The length of the short upward diagonal lines multiplied by a proportionality factor is inversely related to the largest positive Lyapunov exponent (Eckmann et al. 1987). Horizontal and vertical lines result from isolated vectors matching closely with a repeated string of vectors repeated in time. Bands of white space in recurrence plots are due to the presence of transients in the time series. Transients are defined as events that lie far outside the normal distribution of values for the otherwise stationary dynamics. Nonuniform texture or paling of the recurrence plot away from the central diagonal line reflects drift in the system.

Figs. 3 to 6 show the recurrence plot of the 4 quantities with a radius of 5 , an embedding dimension of 10 , and a phase lag of 6 time steps ( $3 \mathrm{wk}$ ) according to the autocorrelation, and Fig. 7 shows the corresponding histograms of the recurrence. The recurrence plots are symmetrical to the main diagonal indicating the identity $(i=j)$ in the distance matrix. Therefore, the upper and lower triangles contain the same information. The intensity of darkness is a measure of the recurrence in the system. Picophytoplankton (Fig. 3) show a large dark square indicating a selected dominant frequency and the horizontal lines in the upper triangle and vertical lines in the lower triangular represent an isolated vector repeating in time. The nonuniform texture away from the central diagonal line reflects drift in the system. Nanophytoplankton (Fig. 4) is characterized by a couple of frequency bands which are represented by the dark squares along the main diagonal. Similar to picophytoplankton, isolated vectors occur in the RQA of nanophytoplankton. In addition, bands of white space appear between the dark squares or frequency bands representing transient behaviour in the time series. The nonuniform texture parallel to the main axes and away from the main diagonal again indicates a drift in the system. Calanoids (Fig. 5) are characterized by 2 frequency bands ( 2 squares along the main diagonal) and a non-linear interaction between them. Transient behaviour in the time series of calanoids is represented by the bands of white space between the frequency bands. In addition the random scattering in the upper left or lower right corner indicates the existence of high-dimensional stochastic processes. Finally, rotifers (Fig. 6) show 2 dominant frequency bands with a non-linear interaction between them and a strong transient behaviour. Similar to the RQAs of both phytoplankton time series, isolated vectors appear repeating in time.

To quantify the identified frequency band in the RQA, recurrence histograms (the abscissa being scaled by increasing lags, i.e. periods) were generated for the 4 time series (Fig. 7). The histograms show a single peak at 7 wk for picophytoplankton, a small band of periods between 4 and $7 \mathrm{wk}$ and isolated peaks at 5

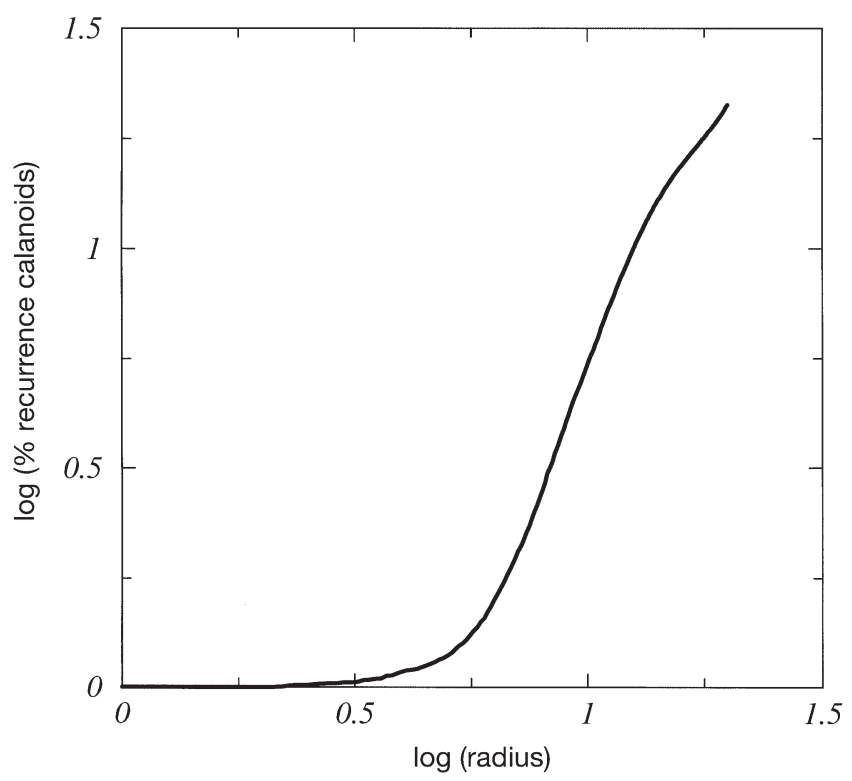

Fig. 2. Logarithm of percent recurrence of calanoids as function of the logarithm of radius 


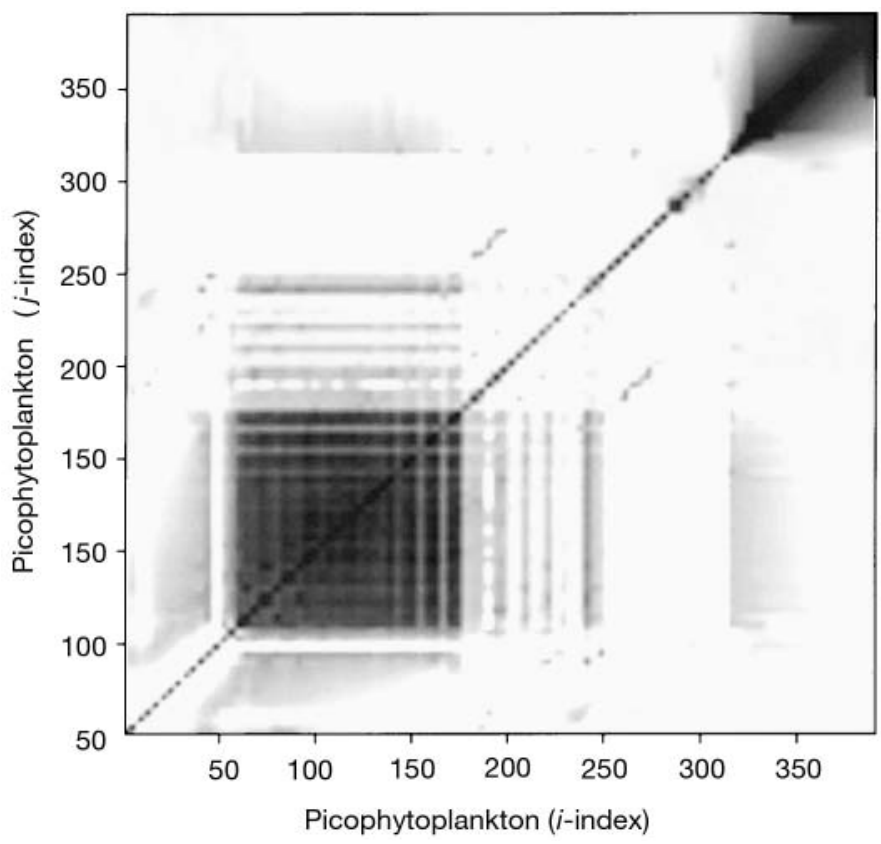

Fig. 3. Recurrence plot for picophytoplankton

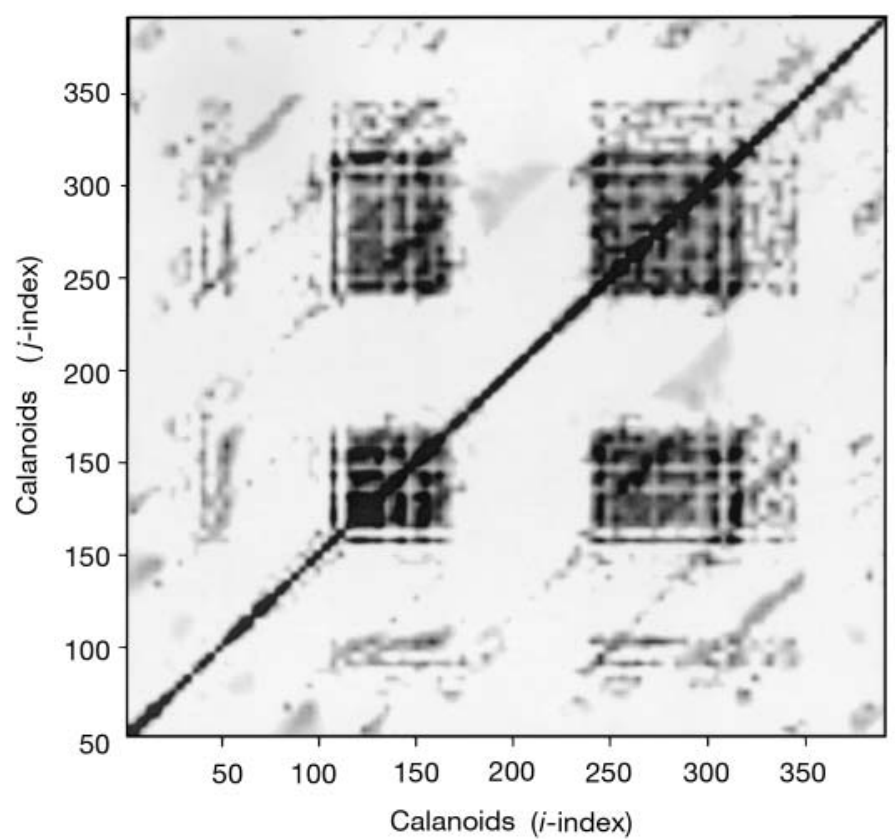

Fig. 5. Recurrence plot for calanoids

and 7 wk for nanophytoplankton. Calanoids have a broad range between 2 and 7 wk with a pronounced peak at $5 \mathrm{wk}$, which fits into the generation time of copopods. In addition 2 minor peaks appear at 3 and 12.5 wk. Rotifers, in contrast, show transient behaviour with an extreme peak at $5.5 \mathrm{wk}$ and a minor peak at 11.5 wk. In contrast to in situ observations, no low fre-

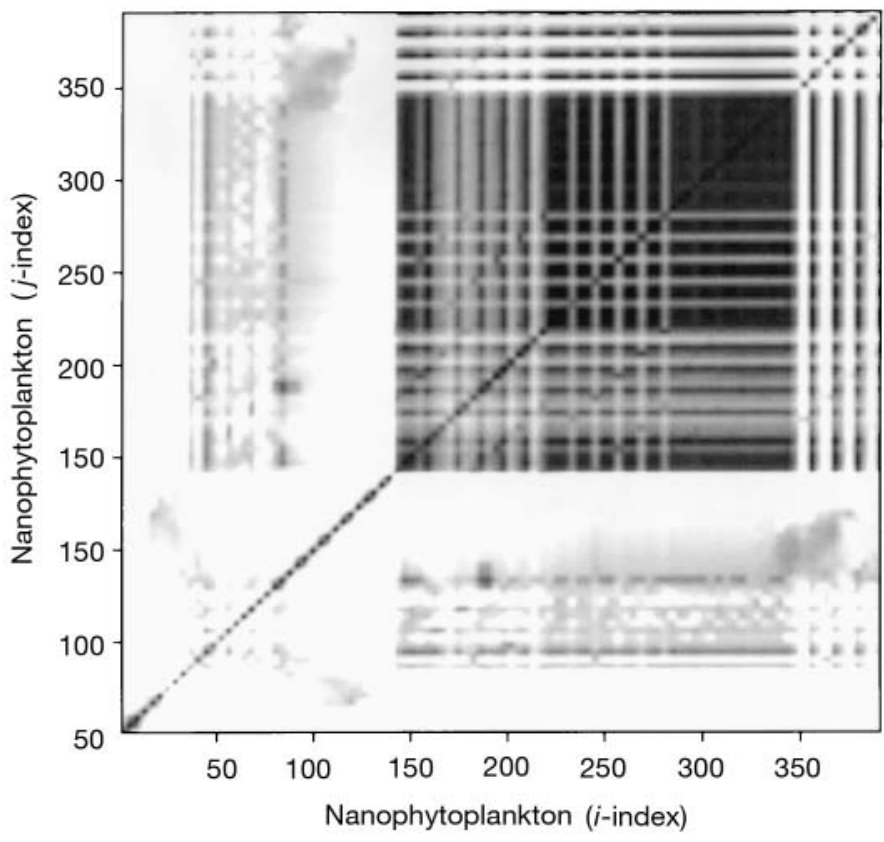

Fig. 4. Recurrence plot for nanophytoplankton

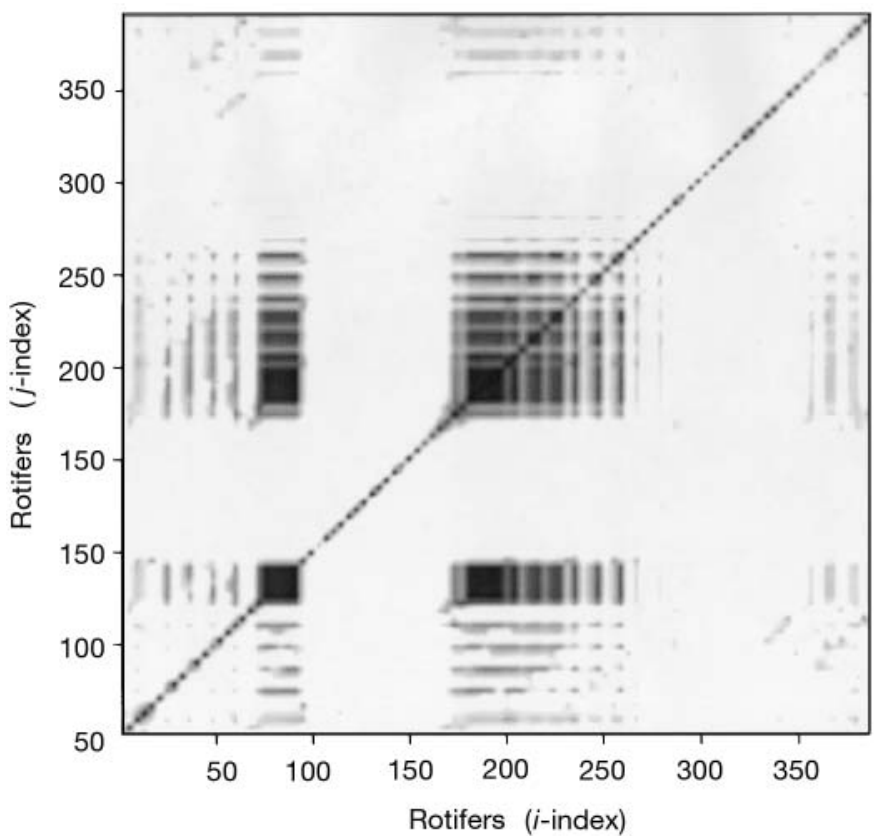

Fig. 6. Recurrence plot for rotifers

quency variability in the mesocosm exists. Hasselmann (1976) has shown that the integration of high frequency noise results in low frequency variability. The zooplankton sampling strategy as well as the removal of data during the reduced light period can be considered as stochastic forcing to the system. The fact that no low frequency variability appears in the mesocosm 
indicates constant experimental conditions, with no influence of the sampling strategy or of the data reduction.

For a quantitative assessment of the nonlinear system dynamics the descriptors of the recurrence plot were compared to the decriptors of a shuffled system (Zbilut \& Webber 1992, Webber \& Zbilut 1994). A comparison of the recurrence of the time series with the shuffled time series, in which the time structure and the phase information are removed, is very useful in understanding the hidden system dynamics, especially for the analysis of possible chaotic behaviour. Table 1 displays the percentage of recurrence, the percentage of determinism, the entropy, the trend and the maximum diagonal line length of the original time series (unshuffled) and the corresponding shuffled time series for picophytoplankton, nanophytoplankton, calanoids and rotifers. A deterministic system with periodic processes is characterized by a high percentage of recurrence, whereas low values indicate more aperiodic processes (Webber \& Zbilut 1994). The absolute values of percentage of recurrence of the shuffled system are relatively high although the phase information is removed. Relatively high values for shuffled determinism are often found for nonstationary dynamics. The reason is straightforward: a trend will decrease the number of recurrences (riding on an increasing/decreasing value), and thus shuffling a trended series 'homogenizes' the series to create more recurrences. Indeed, an inspection of the recurrence plot demonstrates the presence of such a trend. Both the unshuffled and shuffled time series indicate a periodic system dynamics which is not immediately visible from the original time series.

The percent determinism quantifies the percentage of recurrence points that form upward diagonal line segments. This quantity is very important in detecting derterminism in the system if the radius, the time lag and the embedding dimension are chosen correctly. The fact that in all phytoplankton and zooplankton time series the percent determinism of the RQA is very high in the unshuffled situation and decreases tremendously in the shuffled version indicates a fully deterministic system. Entropy is a measure of the complexity of the sytems. In all cases, the RQA of the unshuffled system shows a higher complexity than in the shuffled system. Relative to the shuffled system the complexity is a bit higher in the zooplankton time series than in the phytoplankton. The trend addresses the non-stationarity characteristics in recurrence plots and is a measure of the possible drift in the system. The drift is computed relative to the numbers of recurrent
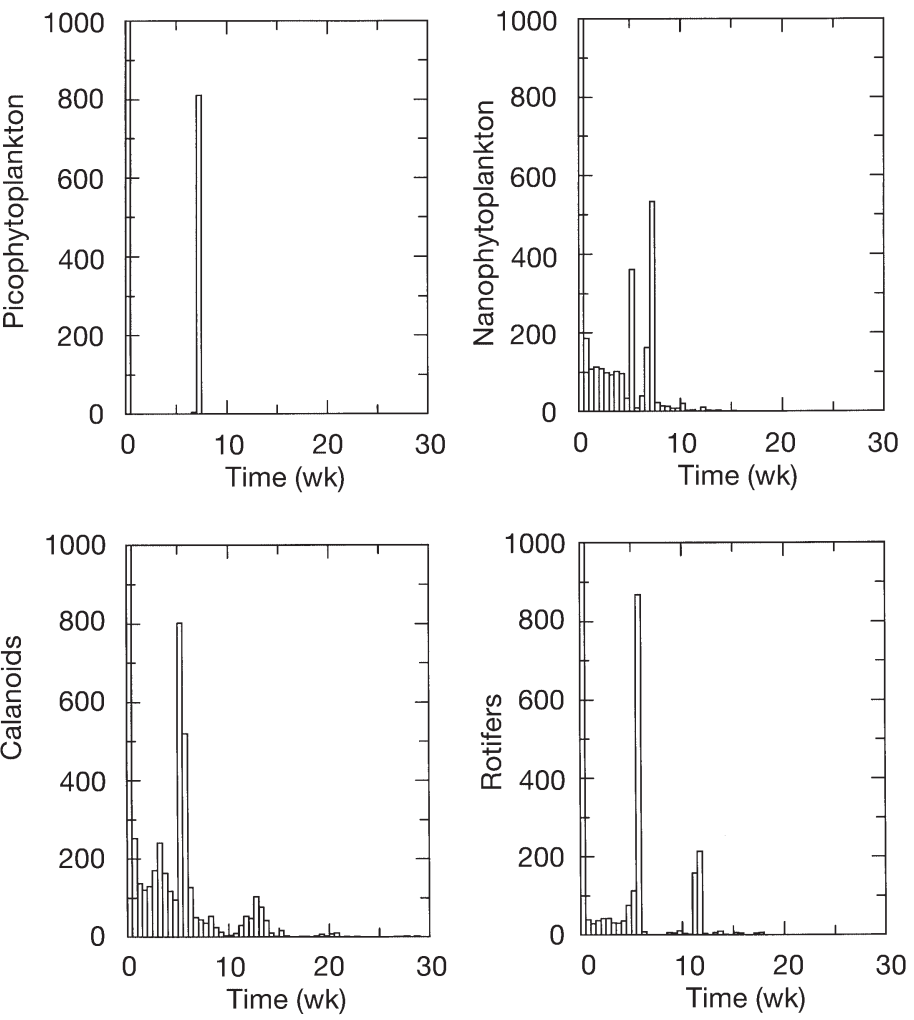

Fig. 7. Recurrence histogram of picophytoplankton, nanophytoplankton, calanoids and rotifers

points extending from the main diagonal to the corner of the plot. The slope of the least square fit is the trend of the system which can have positive or negative values. Sytems without drift have values close to 0. All 4 variables show a negative trend with very high values in phytoplankton and smaller values in zooplankton. An explanation of the trend in phytoplankton might be a change in the composition towards the end of the experiment. The last quantity is the maximum line length ml. Eckmann et al. (1987) have shown that the length of the diagonal lines times a proportionality factor is inversely related to the largest positive Lyapunov exponent $\Lambda$, a measure of the deterministic chaos: $\Lambda^{-1} \propto$ $m l_{\mathrm{u}}-m l_{\mathrm{s}}$. The indices $\mathrm{u}$, s denote unshuffled and shuffled values. The extremely large difference in unshuffled compared to shuffled maximum line length suggests that no deterministic chaos exist in the mesocosm. Indeed, the relatively long line length of 204 (within the limits of computational error) raises the question of whether a positive Lyapunov exponent exists. The same procedure of RQA has been applied to the time coefficients of the 4 EOFs which have a nearly equal distribution of explained variance. The RQA patterns and the system properties are more or less identical and, therefore, they are not shown here. 
Table 1. System properties derived from RQA for the unshuffled and shuffled time series of picophytoplankton, nanophytoplankton, calanoids, and rotifers. Percent recurrence quantifies the percentage of the plot occupied by recurrent points. Percent determinism quantifies the percentage of recurrent points that form upward diagonal line segments. Entropy (in bits per bin) is a measure for the complexity of recurrence plot. The trend addresses the non-stationarity characteristics in recurrence plots and maxline is the length of the longest upward diagonal line way from the main diagonal. Details are given in the section 'Materials and methods: recurrence quantification'

\begin{tabular}{|c|c|c|}
\hline & Unshuffled & Shuffled \\
\hline \multicolumn{3}{|l|}{ Picophytoplankton } \\
\hline$\%$ recurrence & 38.7 & 32.1 \\
\hline$\%$ determinism & 86.8 & 40.3 \\
\hline Entropy & 2.48 & 1.25 \\
\hline Trend & -190.3 & 2.7 \\
\hline maxline & 175 & 13 \\
\hline \multicolumn{3}{|c|}{ Nanophytoplankton } \\
\hline$\%$ recurrence & 45.4 & 32.1 \\
\hline$\%$ determinism & 97.7 & 33.5 \\
\hline Entropy & 2.79 & 1.36 \\
\hline Trend & -149.0 & 14.8 \\
\hline maxline & 204 & 19 \\
\hline \multicolumn{3}{|l|}{ Calanoids } \\
\hline \% recurrence & 23.2 & 18.3 \\
\hline$\%$ determinism & 85.0 & 28.9 \\
\hline Entropy & 2.65 & 0.77 \\
\hline Trend & -81.0 & -0.9 \\
\hline maxline & 83 & 5 \\
\hline \multicolumn{3}{|l|}{ Rotifers } \\
\hline$\%$ recurrence & 27.2 & 24.0 \\
\hline$\%$ determinism & 87.6 & 46.0 \\
\hline Entropy & 2.44 & 0.63 \\
\hline Trend & -28.9 & -21.6 \\
\hline maxline & 101 & 5 \\
\hline
\end{tabular}

\section{DISCUSSION}

The recurrence histograms of the time series reflect the frequency information of RQA in a quantitative way. The additional information on transients, isolated vectors, high-dimensional stochastic processes or drift in the system dynamics cannot be obtained with histogram analysis alone. The combination of both RQA and histogram analysis gives a much better insight into the non-linear system dynamics of mesocosms. The pictures of the histograms hint to rhythmic pattern in the temporal sequence of the peaks. This coincides with results from a statistical time series analysis, where the pictures of auto- and crosscorrelations show oscillatory behaviour.

Multi-species models in theoretical ecology have provided the basis of the theory of deterministic chaos since May \& Oster (1976). The occurrence of chaos in models has attracted much attention during the last 2 decades. The detection of chaos for a given system can be significant from a modelling point of view; low dimensional chaos implies very few degrees of freedom. However, the occurrence of chaos in real world communities is not evident so far. Experiments with plankton mesocosms are a useful approach in this context. It is possible to run them under constant external conditions, which corresponds to the confinement of the models to within-community interactions. Our first trial to search for chaos in a dataset of the mesocosm experiment of Heerkloss \& Klinkenberg (1998) was without success. No chaotic attractor was detected in the time series of the 4 most important groups of the plankton community. The temporal sequence of biomass peaks contained only an oscillating mode of dynamics, although high variability was observed with respect to peak size as well as pattern of temporal sequence. This result may be considered not to be sufficient for the final conclusion that chaos is absent. A chaotic pattern may be detectable if the interaction between planktonic groups is considered in the analysis instead of the species time series itself. This will be done in the future with a more comprehensive dataset of the experiment, which is now available. Using, for example, a 'cross recurrence' method, competition between copepods and rotifers can be investigated. The results of the investigation presented here show that RQA is easy to apply and a helpful mathematical tool for the analysis of time series in ecology.

With the help of RQA we obtained quantitative results for several variables, which allow a comprehensive description of the non-linear behaviour of the system. This may be useful for comparisons between different systems in general, not only with respect to the problem of identifying chaotic behaviour but also studying deterministic behaviour, transient processes, and trends in biological time series. For example, the relation between species diversity and non-linear dynamics of plankton communities may be a research field where RQA could be applied. The competition models of Huisman \& Weissing (1999) show that the number of species carried in a community increases with an increase in amplitude of biomass fluctuation. Data created by different models could be analyzed and described by RQA in order to quantify the relationship between diversity and variability. RQA could also be a useful tool if multispecies models are adjusted to datasets from empirically measured time series.

Acknowledgements. The authors are indebted to Alessandro Giuliani for helpful comments and discussions. J. Dippner was supported by mare Dreiviertel Verlag, which is greatly acknowledged. 


\section{LITERATURE CITED}

Allen JC (1993) Chaos reduces species extinction by amplifying local population noise. Nature 364:229-232

Ascioti FA, Beltrami E, Carroll TO, Wirick C (1993) Is there chaos in plankton dynamics? J Plankton Res 15:603-617

Bergelson V (2000) The multivarious Poincaré recurrence theorem. In: Foreman M (ed) Descriptive set theory and dynamical systems. Cambridge University Press, Cambridge, p 31-57

Doveri F, Scheffer M, Rinaldi S, Muratori S, Kuznetsov Y (1993) Seasonality and chaos in a plankton-fish-model. Theor Popul Biol 43:159-183

Eckmann JP, Kamphorst SO, Ruelle D (1987) Recurrence plot of dynamical systems. Europhys Lett 4:324-327

Feller W (1968) An introduction to probability theory and its applications, Vol 1. Wiley, New York, p 303-341

Gao JB (1999) Recurrence time statistics for chaotic systems and their applications. Phys Rev Lett 83:3178-3181

Giuliani A, Piccirillo G, Marigliano V, Colosimo A (1998a) A nonlinear explanation of aging-induced changes in heartbeat dynamics. Am J Physiol 275 (Heart Circ Physiol 44): H1455-H1461

Giuliani A, Colosimo A, Benigni R, Zbilut JP (1998b) On the constructive role of noise in spatial systems. Phys Lett A 247:47-52

Giuliani A, Benigni R, Sirabella P, Zbilut JP, Colosimo A (2000) Nonlinear methods in the analysis of protein sequences: a case study in rubredoxins. Biophys J 78: $136-148$

Hasselmann K (1976) Stochastic climate models. Part I. Theory. Tellus 28:473-485

Hastings A, Powell T (1991) Chaos in a 3-species food chain. Ecology 73:896-903

Hastings A, Horn CL, Ellner S, Turchin P, Godfray HCJ (1993) Chaos in ecology: is mother nature a strange attractor? Annu Rev Ecol Syst 25:1-33

Heerkloss R, Klinkenberg G (1998) A long-term series of a planktonic foodweb: a case of chaotic dynamics. Verh Int Ver Limnol 26:1952-1956

Hegger R, Kantz L, Matassimi L, Schreiber T (2000) Coping with nonstationarity by overembedding. Phys Rev Lett 84: 4092-4095

Huisman J, Weissing FJ (1999) Biodiversity of plankton by species oscillations and chaos. Nature 402:407-410

Hutchinson GE (1961) The paradox of the plankton. Am Nat 95:137-145

Jenkins GM, Watts DG (1968) Spectral analysis and its applications. Holden Day, San Francisco, CA

Joergensen SE (1997) Integration of ecosystem theory: a pattern. Kluwer Academic Publishers, Dordrecht

Manetti C, Ceruso MA, Giuliani A, Webber CL Jr, Zbilut JP

Editorial responsibility: Otto Kinne (Editor),

Oldendorf/Luhe, Germany
(1999) Recurrence quantification analysis as a tool for characterization of molecular dynamics simulations. Phys Rev E 59:992-998

May RM, Oster GF (1976) Bifurcation and dynamic complexity in simple ecological models. Am Nat 110:573-599

McCann K, Yodzis P (1994) Biological conditions for chaos in a three-species food chain. Ecology 75:561-564

Rinaldi S, Solidoro C (1998) Chaos and peak-to-peak dynamics in a plankton-fish model. Theor Popul Biol 54:62-77

Schaffer M (1991) Should we expect strange attractors behind plankton dynamics - and if so should we bother? J Plankton Res 13:1291-1305

Schaffer WM, Kot M (1985) Do stange attractors govern ecological systems? BioScience 35:342-350

Schuster HG (1989) Deterministic chaos. An introduction. VHC Verlagsgesellschaft, Weinheim

Shannon CE, Weaver W (1949) The mathematical theory of information. University of Illinois Press, Urbana

Solé RV, Valls J (1992) Spiral waves, chaos and multiple attractors in lattice models of interacting populations. Phys Lett A 166:123-128

Trulla LL, Giuliani A, Zbilut JP, Webber CL Jr (1996) Recurrence quantification analysis of the logistic equation with transients. Phys Lett A 223:225-260

Turchin P (1993) Chaos and stability in rodent population dynamics: evidence from nonlinear time-series analysis. Oikos 68:167-172

Webber CL Jr, Zbilut JP (1994) Dynamical assessment of physiological systems and states using recurrence plot strategies. J Appl Physiol 76:965-973

Zbilut JP, Webber CL Jr (1992) Embeddings and dalays as derived from quantification of recurrence plots. Phys Lett A 171:199-203

Zbilut JP, Koebbe M, Mayer-Kress G (1992) Use of recurrence plots in the analysis of heart beat intervals. In: Proceedings computers in cardiology, IEEE Computer Society Press, Los Alamitos, p 263-266

Zbilut JP, Giuliani A, Webber CL Jr, Colosimo A (1998a) Recurrence quantification analysis in structure-function relationship of proteins: an overview of a general methodology applied to the sace of TEM-1 $\beta$-lactamase. Protein Eng 11:101-107

Zbilut JP, Giuliani A, Webber CL Jr (1998b) Recurrence quantification analysis and principal components in the detection of short complex signals. Phys Lett A 237:131-135

Zbilut JP, Giuliani A, Webber CL Jr (1998c) Detecting deterministic signals in exceptionally noisy environments using cross-recurrence quantification. Phys Lett A 246:122-128

Zbilut JP, Giuliani A, Webber CL Jr (2000) Recurrence quantification analysis as an empirical test to distinguish relatively short deterministic versus random number series. Phys Lett A 267:174-178

Submitted: April 20, 2001; Accepted: April 15, 2002 Proofs received from author(s): September 12, 2002 